\title{
KINETOPLASTIDA: NEW THERAPEUTIC STRATEGIES
}

\author{
CROFT S.L.*
}

\section{Summary :}

New formulations and therapeutic switching of the established drugs, amphotericin B and paromomycin, together with the discovery of miltefosine, have significantly improved the opportunities for treatment of visceral leishmaniasis (VL) chemotherapy. However, for human African trypanosomiasis $(H A T)$, Chagas disease and cutaneous leishmaniases there has been limited progress. For HAT, a novel diamidine, parfuramidine, is in phase III clinical trial for early-stage disease, but for the treatment of late-stage disease there are no new drugs and combinations of eflornithine with melarsoprol or nifurtimox have been the focus of clinical studies. For Chagas disease, different classes of compounds that have validated biochemical targets, sterol biosynthesis methylases and cysteine proteases, are in various stages of development. The genome sequences that are now available for the pathogens that cause the leishmaniases and trypanosomiases, and new methods for rapid validation of targets, are part of the solution to discover new drugs. The integration of medicinal chemistry, pharmacokinetics, project planning and interaction with the pharma/biotech sector are essential if progress is to be made. Although there are financial constraints, the appearance of new funding sources and notfor-profit product development partnerships offers hope for drug development.

KEY WORDS : visceral leishmaniasis, cutaneous leishmaniasis, human African trypanosomiasis, Chagas disease, drugs, chemotherapy.

T The drugs used for treatment of the leishmaniases and the trypanosomiases (see Table I) are fraught with problems of toxicity, variable efficacy, parenteral administration or length of treatment. For the trypanosomiases and cutaneous leishmaniasis, there are limited drugs or treatments in clinical development. In contrast, for visceral leishmaniasis there has been some progress with the availability of liposomal amphotericin B, miltefosine and paromomycin for treatment in India. Although considerable advances in the identification, validation and characterization of drug targets has come with the completion of the genomes for Trypanosoma cruzi, Trypanosoma brucei and Leishmania major (Berriman et al., 2005) and new tools such as RNAi (Balana-Fouce \& Reguera, 2007) have been developed, this is only one early part of the long and complex process of drug discovery and development.

\footnotetext{
* Department of Infectious and Tropical Diseases, London School of Hygiene \& Tropical Medicine, London WC1E 7HT, UK.

E-mail: simon.croft@lshtm.ac.uk
}

New and established pharmacophores, based upon synthetic and natural product chemistry, have to be brought into place along with improved screening technologies to identify hits (Frearson et al., 2007). Appropriate models of infection and early pharmacokinetic studies to evaluate leads, also need to be integrated into the process, as seen at academic centres in USA (www.ucsf.edu/mckerrow) and UK (www.dundee.ac.uk/biocentre). The support of public-private partnerships like the Drugs for Neglected Diseases initiative (www.dndi.org) and interaction with the commercial sector is also required. Further development will depend upon the expertise in lead optimization, toxicology and pharmacology available in the pharmaceutical and biotech sectors and CROs with the associated requirement for high level funding (see for example www.dndi.org). Clinical trials capacity is also required and is being addressed in India, Africa (www.sti.ch) and South America. The issues around drug production and delivery also need to be addressed; the example of paromomycin delivery for VL in India provides some model for the future (www.iowh.org).

\section{LEISHMANIASIS}

There have been different rates of development and there are different issues associated with drug development for visceral leishmaniasis (VL) and cutaneous leishmaniasis (CL); these two manifestations will therefore be treated separately.

\section{VISCERAL LEISHMANIASIS}

Pentavalent antimonials, the standards drugs for 60 years, are now almost obsolete in India due to drug resistance (Croft et al., 2006), but are still useful in the rest of the world. The introduction of generic brands has reduced costs. Amphotericin B, normally considered a second line drug, is now first line in Bihar state, India. Although a number of amphotericin B lipid formulations, developed during the 1980s for treatment of systemic mycoses in immunocompromised patients, have proved effective in the treatment of VL, only one of 


\section{Visceral leishmaniasis}

First line drugs

Clinical trials

Cutaneous leishmaniasis

First line drugs

Clinical trials

Human African trypanosomiasis

- Haemolymphatic stage

First line drugs

Clinical Trial

- CNS stage

First line drugs

Clinical Trial

Chagas disease

First line drugs

- Acute stage

- Indeterminate stage

- Chronic stage

Clinical trials
Sodium stibogluconate (Pentostam and SSG)

Meglumine antimoniate (Glucantime)

Amphotericin B (Fungizone)

Liposomal amphotericin B (AmBisome)

Miltefosine

Paromomycin

Sitamaquine

Other amphotericin B formulations

Sodium stibogluconate (Pentostam)

Meglumine antimoniate (Glucantime)

Amphotericin B (Fungizone)

Pentamidine

Paromomycin (topical formulation)

Paromomycin (topical formulations, phases II

and III)

Miltefosine (oral, phase III)

Imiquimod (topical immunomodulator, phase II)

Pentamidine

Suramin

Parfuramidine (DB289)

Melarsoprol

Eflornithine

Nifurtimox and eflornithine co-administration
Generic sodium stibogluconate (SSG) from Albert David (India) has made treatment cheaper. Sanofi-Aventis have also reduced the price of Glucantime

This has proved to be the most effective and least toxic lipid formulation for VL Registered in India and available on private market. Cheaper price available via WHO In phase IV trials in India

Completed phase II trials in India

Further pre-clinical research for development of cheaper formulations

For complex manifestations, like mucosal leishmaniasis

For specific forms in South America

Formulations from WRAIR (Washington) and FioCruz (Belo Horizonte) on trial There appears to be differences in species sensitivity. Registered in Colombia

As an adjunt therapy to antimonials. Reported not to be effective in Iran

Phase III trials in DRC and Sudan

Phase III trial with DNDi and WHO TDR

Table I. - Drugs in use or on clinical trial in 2007.

these, the liposomal formulation AmBisome, has become a standard. It is registered for the treatment of VL in various countries, its use is described by a WHO working group (Bern et al., 2006) and a single dose therapy of $5 \mathrm{mg} / \mathrm{kg}$ has been shown to cure $90 \%$ patients in India (Sundar et al., 2003). A significant reduction in price negotiated by WHO with the producers (Gilead) will have an impact, but AmBisome will remain an expensive treatment. A parenteral formulation of the aminoglycoside paromomycin, has moved slowly through clinical trials over the past decades, more recently showing $94 \%$ efficacy (15 mg/ $\mathrm{kg}$ for 21 days) in phase III clinical trials in India (Sun- dar et al., 2007) and was registered there for VL in 2006. Like paromomycin, the anti-leishmanial activity of the phospholipid derivative, miltefosine (Fig. 1) was first identified at the Wellcome laboratories, UK. (Croft \& Engel, 2006). This drug has provided the first oral treatment for VL and the first to undergo phase IV studies (Bhattacharya et al., 2007).

Other opportunities remain. Rational approaches, pyrazolopyrimidines (allopurinol and derivatives) that disrupt purine-salvage and nucleic acid biosynthesis, and the inhibitors of 14- $\alpha$ demethylase and sterol biosynthesis (antifungal azoles), proved to have disappointing efficacy, due to poor pharmacokinetic properties 
$\mathrm{H}_{3} \mathrm{C}\left(\mathrm{CH}_{214}\right)_{14}-\mathrm{CH}_{2}-\mathrm{O}-\overbrace{\mathrm{O}^{-}}^{\mathrm{O}}-\mathrm{O}-\mathrm{CH}_{2} \mathrm{CH}_{2} \mathrm{~N}^{+}\left(\mathrm{CH}_{3}\right)_{3}$

(b)<smiles>NCCCC(N)(C(=O)O)C(F)F</smiles>

(c)

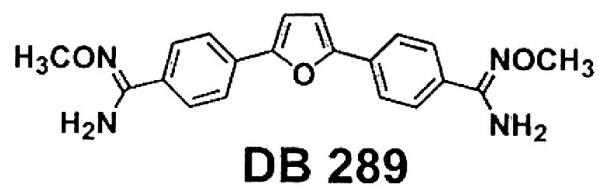

(d)

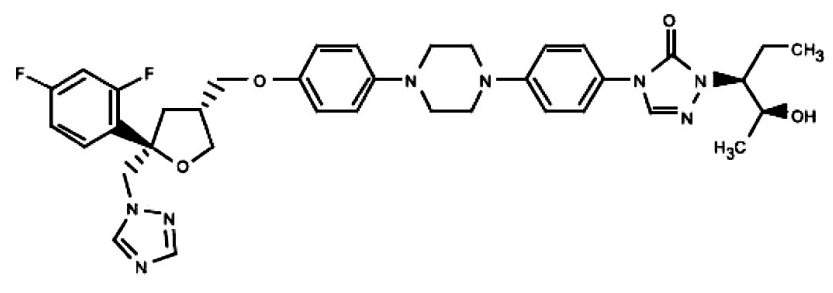

(e)

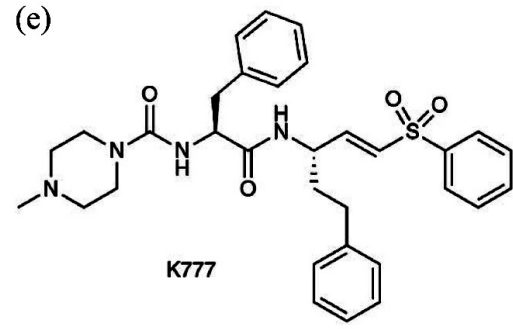

Fig. 1. - New drugs in clinical or pre-clinical development for the trypanosomiases and leishmaniasis

(a) Miltefosine, an alkylphosphocholine, originally developed as an anti-cancer agent, subsequently developed for treatment of both VL and CL by Zentaris AG (Germany) and WHO/TDR.

(b) Eflornithine, difluormethylornithine, an irreversible inhibitor of ornithine decarboxylase and polyamine biosynthesis, originally developed as an anti-cancer agent, but used for the treatment of CNS stage T. $b$. gambiense infection.

(c) DB 289, a diamidine, pro-drug of 2,5-bis(4-amidinophenyl)furan (DB75 or furamidine), currently in phase III clinical trials for treatment of haemolymphatic stage T. b. gambiense infection in central Africa, in development with N. Carolina University Gates Consortium.

(d) Posaconazole, a triazole derivative, that inhibits Trypanosoma cruzi sterol C14 $\alpha$ sterol demethylase and can eradicate the parasite from animal models of both acute and chronic Chagas disease. Registered as a systemic antifungal agent.

(e) K777 (N-methyl-piperazine-urea-F-hF-vinyl-sulfone-phenyl), a specific inhibitor of cruzipain, an essential cysteine protease of Trypanosoma cruzi, with trypanocidal activity, both in vitro and in vivo.
(Shapiro et al., 1991) or biochemical routes permitting bypass in intracellular parasites (Roberts et al., 2003). However, allopurinol remains a component of treatment of canine VL and the triazole posoconazole was effective in rodent models (Al-Abdely et al., 1999). Natural product screens have identified chalcones (Zhai et al., 1999), maesabalides (Maes et al., 2004), and novel quinolines (Nakayama et al., 2006) with activity in rodent VL models, although metabolic and toxic liabilities have limited the progress of lead compounds. Other opportunities lie in understanding of metabolic pathways and enzyme targets, with studies on isoprenoid biosynthesis leading to studies on bisphosphonates (Yardley et al., 2002), and on kinases to paullones (Grant et al., 2004).

\section{CuTANEOUS LEISHMANIASIS (CL)}

In comparison to VL there are limited proven options for CL (see Table I). Pentavalent antimonials have proved inconsistent in their effectiveness across the different Leishmania species (Croft et al., 2006), and pentamidine and amphotericin $\mathrm{B}$ are limited to specific types of CL (see Alvar et al., 2006). Paromomycin in various topical formulations has variable efficacy (see Garnier \& Croft, 2002), and there is a continuing search for more effective and less irritant topical creams and gels (Ben-Salah et al., 2005). Oral miltefosine also has some variable, species dependent effectiveness against CL (Soto et al., 2004: Yardley et al., 2005) and is now registered for this indication in Colombia (2005). Further studies are required to define effectiveness against different forms of this disease.

\section{HUMAN AFRICAN TRYPANOSOMIASIS}

ignificant advances in our understanding of the S biology of Trypanosoma brucei have not yet led to new drugs (Berriman et al., 2005). Since the registration of the ornithine decarboxylase inhibitor, eflornithine, in 1990 for late stage gambiense disease (Burri \& Brun, 2003) no novel treatments have been introduced. The lower incidence and severity of adverse effects of eflornithine when compared to melarsoprol, has led some to advocate that this drug should become the first line treatment for late stage HAT (Chappuis et al., 2005). The requirement for high doses and prolonged intravenous infusion, however make the drug expensive and difficult to distribute and administer in rural Africa. Its availability as a trypanocide is dependent upon commitments made to MSF and WHO by the manufacturing company (Sanofi-Aventis). There have been other approaches to improve the use of currently registered drugs. Pharmacokinetic studies of melarsoprol led to the successful testing of a shortened 
10 day course (rather than 21-35 days) which improves patient compliance and reduces hospital costs (Schmidt et al., 2004). Studies aimed to modify dosing with eflornithine are also underway with clinical studies on coadministrations with melarsprol or nifurtimox recently reported. These studies are essential in the face of the increased incidence of treatment failure with melarsoprol in some HAT foci (Brun et al., 2001). Field studies to determine the cause of these failures are of importance and are in progress.

There is only one drug currently in clinical trials. The orally available prodrug, parfuramidine (DB289) (Fig. 1), is converted systemically into another diamidine (furamidine, DB75) that is active against early stage disease (Ansede et al., 2004). The blood brain barrier remains a challenge in drug design to ensure sufficient drugs reaches parasites within the brain of late stage patients. A large number of diamidines have been synthesized through a consortium led by the University of North Carolina (funded by the Bill and Melinda Gates Foundation) and other pro-drugs from the same series active against late stage disease have emerged. Trypanosomes are highly sensitive to selected nitroheterocyclic compounds that have shown activity against CNS stage infections in experimental models. Although clinical studies have suggested that the nitrofuran nifurtimox is insufficiently active alone for treatment of HAT, studies have ensured that this drug is tested in combination (ibid) and that other potent and less genotoxic compounds are sought (Stewart et al., 2004 and www.dndi.org).

Research on unique metabolic targets in trypanosomes (Berriman et al., 2005), including that around thiol metabolism, in particular on trypanothione reductase, on energy metabolism, in particular on glycosomal enzymes, and on polyamine metabolism, with interesting novel compounds identified but without any clear candidates for lead optimization emerging (see Luscher et al., 2007). The lack of advances in treatment for HAT highlight the need for closer integration of chemistry and biology efforts and improved understanding of the pharmacokinetic and pharmacodynamic requirements of the ideal drug.

\section{SOUTH AMERICAN TRYPANOSOMIASIS (CHAGAS DISEASE)}

S imilar to above, despite the impressive advances in our knowledge about the biology of T. cruzi (El-Sayed et al., 2005), the only drugs currently available are the nitrofuran nifurtimox and the 2-benzimidazole benznidazole, which were developed 1960's and 1970's. These drugs are active in the acute stage of the disease (up to $80 \%$ efficacy) but of limited effi- cacy against established chronic stage disease, require long courses of treatment (60 days) and have severe side effects. With the reduction of transmission of Chagas disease in several foci in S. America, there has been greater focus on the needs for treatment of indeterminate and early chronic phases. Thorough experimental studies show that persistence of parasites, coupled with an imbalanced immune response that could include autoimmune reactions, generate sustained inflammatory responses in infected tissues producing the characteristic lesions of chronic Chagas disease (Tarleton et al., 2001). Significant reduction of T. cruzi from infected patients appears, therefore, to be essential to prevent disease progression and to avert its irreversible longterm consequences. Studies with benznidazole have shown that it has some efficacy in early chronic infections (Sosa-Estani et al., 1998), and a long term clinical trial (BENEFIT) is now underway to determine the extent of use of this drug for this indication (http://clinicaltrials.gov/show/NCT00123916). There are several rational approaches to the treatment of Chagas disease that have identified novel compounds or the potential for therapeutic switching. The potential of specific ergosterol biosynthesis inhibitors that act at the level of $\mathrm{C} 14 \alpha$ sterol demethylase, has long been known, and some like ketoconazole entered clinical studies decades ago. However, studies by Urbina and colleagues have shown that new anti-fungal triazole derivatives (Fig. 1), for example posoconazole, have very high potency against T. cruzi, and are capable of curing chronic infections in mice (Molina et al., 2000). One of this drug class will certainly enter clinical trials. Other novel ergosterol biosynthesis inhibitors that act at the level of squalene synthase or oxidosqualene cyclase (Urbina \& Docampo, 2003) also show potential. Inhibitors of cruzipain, an essential protease specific to the parasite (Cazzulo et al., 2002) and one particular vinyl sulphone, $\mathrm{K} 777$, is in pre-clinical development (Doyle et al., 2007). Other studies have identified inhibitors of targets on the isoprenoid biosynthesis pathway, including farnesyl transferase and farnesyl pyrophosphate synthase. N-alkyl-bisphosphonates, inhibitors of farnesyl that selectively accumulate in the parasite's acidocalcisomes, also have activity in experimental models (Garzoni et al., 2004).

\section{DISCUSSION}

Whe recent publication of the genome sequences of the pathogens that cause leishmaniasis and trypanosomiasis helps to identify both similarities as well as differences in potential drug targets. The subtle differences between the parasites in their metabolic adaptations, the required pharmacokinetic properties of drugs for the different sites of infection, and 
the different approaches required for acute and chronic infections, indicates that we are not in the business of discovering one drug. Each of these three diseases will require several drugs or formulations of drugs for the treatment of all their manifestations. The discovery and development of these new drugs will require: $i$ ) increased input from the disciplines of chemistry, pharmacology, toxicology and pharmaceutics, and ii) further development of suitable disease models and methods for progressing leads and candidate drugs through pre-clinical studies. The limited progress in drug development of the past decades is part of history. Changes in donor patterns, in incentives in the new not-forprofit model of drug development, in the engagement of the pharmaceutical industry bode well for the future. But this should not be taken for granted and the responsibility for a sustained effort in this field requires effective teams, prioritization where necessary and decisions.

\section{REFERENCES}

Al-Abdely H.M., Graybill J.R., Loebenberg D. \& Melby P.C. Efficacy of the triazole SCH 56592 against Leishmania amazonensis and Leishmania donovani in experimental murine cutaneous and visceral leishmaniases. Antimicrobial Agents and Chemotherapy, 1999, 43, 2910-2914.

Ansede J.H., Anbazhagan M., Brun R., Easterbrook J.D., hall J.E. \& Boykin D.W. O-alkoxyamidine prodrugs of furamidine: in vitro transport and microsomal metabolism as indicators of in vivo efficacy in a mouse model of Trypanosoma brucei rhodesiense infection. Journal of Medicinal Chemistry, 2004, 47, 4335-4338.

Balana-Fouce R. \&, Reguera R.M. RNA interference in Trypanosoma brucei: a high-throughput engine for functional genomics in trypanosomatids? Trends in Parasitology, 2007, 23, 348-351.

Bhattacharya S.K., Sinha P.K., Sundar S., Thakur C.P., JHA T.K., Pandey K., Das V.R., Kumar N., Lal C., Verma N., Singh V.P., Anders G., Sindermann H. \& Ganguly N.K. Phase 4 trial of miltefosine for the treatment of Indian visceral leishmaniasis. Journal of Infectious Diseases, 2007, 196, 591-598.

BEN SALAH A. et al. WR279396 an efficient non toxic topical treatment of old world cutaneous leishmaniosis. Abstract book - Third World Congress on Leishmaniosis - April 2005, Sicily, Italy.

Bern C., Adler-Moore J., Berenguer J., Boelaert M., den Boer M., Davidson R.N., Figueras C., Gradoni L., Kafetzis D.A., Ritmeijer K., Rosenthal E., Royce C., Russo R., Sundar S. \& Alvar A. Liposomal amphotericin B for the treatment of visceral leishmaniasis. Clinical Infectious Diseases, 2006, 43, 917-924

Brun R., Schumacher R., Schmid C., Kunz C. \& Burri C. The phenomenon of treatment failures in human African trypanosomiasis. Tropical Medicine \& International Health, 2001, 6, 906-914.
Berriman $\mathrm{M}$ et al. The genome of the African trypanosome Trypanosoma brucei. Science, 2005, 309, 416-422.

BurRI C. \& BRUN R. Eflornithine for the treatment of human African trypanosomiasis. Parasitology Research, 2003, 90 (Supp. 1), S49-S52.

Cazzulo J.J. Proteinases of Trypanosoma cruzi: patential targets for the chemotherapy of Chagas disease. Current Topics Medicinal Chemistry, 2002, 2, 1261-1271.

Chappuis F., Udayraj N., Stietenroth K., Meussen A. \& Bovier P.A. Eflornithine is safer than melarsoprol for the treatment of the second stage Trypanosoma brucei gambiense human African trypanosomiasis. Clinical Infectious Diseases, 2005, 41, 748-751.

Croft S.L. \& Engel J. Miltefosine-discovery of the antileishmanial activity of phospholipid derivatives. Transactions of the Royal Society of Tropical Medicine, 2006, 100, S4s8.

Croft S.L., Sundar S. \& Fairlamb A.H. Drug resistance in leishmaniasis. Clinical Microbiological Reviews, 2006, 19, 111126.

Doyle P.S., Zhou Y.M., Engel J.C. \& McKerrow J.H. A cysteine protease inhibitor cures Chagas' disease in an immunodeficient-moiuse model of infection. Antimicrobial Agents and Chemotherapy, 2007, 51, 3932-3939.

EL-SAYED N.M. et al. Comparative genomics of trypanosomatid parasitic protozoa. Science. 2005, 309, 404-409.

Frearson J.A., Wyatt P.G., Gilbert I.H. \& Fairlamb A.H. Target assessment for antiparasitic drug discovery. Trends in Parasitology, 2007, 23, 589-595.

Garzoni L.R., Waghabi M.C., Baptista M.M., De Castro S.L., Meirelles Mde N., Britto C.C., Docampo R., Oldfield E. \& URBINA J.A. Antiparasitic activity of risedronate in a murine model of acute Chagas' disease. International Journal of Antimicrobial Agents, 2004, 23, 286-290.

Grant K.M., Dunion M.H., Yardley V., Skaltsounis A.L., Marko D., Eisenbrand G., Croft S.L., Meijer L. \& Mottram J.C. Inhibitors of Leishmania mexicina CRK3 cyclin-dependent kinase: chemical library screen and antileishmanial activity. Antimicrobial Agents and Chemotherapy, 2004, 48, 3033-3042.

Luscher A., De Koning H.P. \& MASER P. Chemotherapeutic strategies against Trypanosoma brucei; drug targets vs. drug targeting. Current Pharmaceutical Research, 2007, 13, 555-567.

Maes L., Germonprez N., Quirijnen L., Van Puyvelde L., Cos P. \& VANDEN BERGHE D. Comparative activities of the triterpene saponin maesabalide III and liposomal amphotericin B (AmBisome) against Leishmania donovani in hamsters. Antimicrobial Agents and Chemotherapy, 2004, 48, 2056-2060.

Molina J., Martino-Filho O., Brener Z., Romanha A.J., LoeBENBERG D. \& URBINA J.A. Activities of the triazole SCH 56592 (posaconazole) against drug-resistant strains of the protozoan parasite Trypanosoma (Schizotrypanum) cruzi in immunocompetent and immunosuppressed murine hosts. Antimicrobial Agents and Chemotherapy, 2000, 44, 150-155.

Nakayama H., Loiseau P.M., Bories C., Torres de Ortiz S., Schinini A., Serna E., Rojas de Arias A., FaKhfakh M.A., Franck X., Figadere B., HocQuemiller R. \& Fournet A. Efficacy of 
orally administered 2-substituted quinolines in experimental murine cutaneous and visceral leishmaniases. Antimicrobial Agents and Chemotherapy, 2005, 49, 4950-4956.

Roberts C.W., McCleod R., Rice D.W., Ginger M., Chance M.L. \& GOAD L.J. Fatty acid and sterol metabolism: potential antimicrobial targets in apicomplexan and trypanosomatid parasitic protozoa. Molecular and Biochemical Parasitololgy, 2003, 126, 129-142.

Schmid C., Nkunku S., Merolle A., Vounatosu P. \& Burri C. Efficacy of 10-day melarsoprol schedule two years after treatment for late-stage gambiense sleeping sickness. Lancet, 2004, 364, 789-790.

Sosa-Estani S., Segura E.L., Ruiz A.M., Velazquez E., Porcel B.M. \& YAMPOTIS C. Efficacy of chemotherapy with benznidazole in children in the indeterminate phase of Chagas' disease. American Journal of Tropical Medicine and Hygiene, 1998, 59, 526-529.

Shapiro T.A., Were J.B., Dauso K., Nelson D.J., Desjardins R.E. \& Pamplin C.L. Pharmacokinetics and metabolism of allopurinol riboside. Clinical Pharmacology Therapeutics, 1991, 49, 506-514.

Soto J., Arana B.A., Toledo J., Rizzo N., Vega J.C., Diaz A., Luz M., Gutierrez P., Arboleda M., Berman J.D., Junge K., Engel J. \& Sindermann H. Miltefosine for new world cutaneous leishmaniasis. Clinical Infectious Diseases, 2004, 38, 1266-1272.

Stewart M.L., Bueno G.J., Baliani A., Klenke B., Brun R., Brock J.M., Gilbert I.H. \& BarretT M.P. Trypanocidal activity of melamine-based nitroheterocycles. Antimicrobial Agents and Chemotherapy, 2004, 48, 1733-1738.

Sundar S., Jha T.K., Thalur C.P., Mishra M., Singh V.P. \& BufFELS R. Single-dose liposomal amphotericin B in the treatment of visceral leishmaniasis in India: a multicenter study. Clinical Infectious Diseases, 2003, 37, 800-804.

Sundar S., Jha T.K., Thakur C.P., Sinha P.K. \& BhatTacharya S.K. Injectable paromomycin for visceral leishmaniasis in India. New England Journal of Medicine, 2007, 356, 25712578.

TARLETON R.L. Parasite persistence in the aetiology of Chagas disease. International Journal for Parasitology, 2001, 31, 550-554.

Urbina J.A. \& Docampo R. Specific chemotherapy of Chagas disease: controversies and advances. Trends in Parasitology, 2003, 19, 495-501.

Yardley V., Croft S.L., De Doncker S., Dujardin J.C., Koirala S., Rijal S., Miranda C., Llanos-Cuentas A. \& Chappuis F. The sensitivity of clinical isolates of Leishmania from Peru and Nepal to miltefosine. American Journal of Tropical Medicine and Hygiene, 2005, 73, 272-275.

Yardley V., Khan A.A., Martin M.B., Slifer T.R., Araujo F.G., Moreno S.N.J., Docampo R., Croft S.L. \& Oldfield E. In vivo activity of the farnesyl pyrophosphate inhibitors aledronate, pamidronate and risedronate against Leishmania donovani and Toxoplasma gondii. Antimicrobial Agents and Chemotherapy, 2002, 46, 929-931.

Zhai L., Chen M., Blom J., Theander T.G., Christensen S.B. \& Kharazmi A. The antileishmanial activity of novel oxygenated chalcones and their mechanism of action. Journal of Antimicrobial Chemotherapy, 1999, 43, 793-803. 